\title{
On the Accepting Power of 2-Tape Büchi Automata
}

\author{
Olivier Finkel \\ Equipe de Logique Mathématique \\ U.F.R. de Mathématiques, Université Paris 7 \\ 2 Place Jussieu 75251 Paris cedex 05, France \\ finkel@logique.jussieu.fr.
}

\begin{abstract}
We show that, from a topological point of view, 2-tape Büchi automata have the same accepting power than Turing machines equipped with a Büchi acceptance condition. In particular, for every non null recursive ordinal $\alpha$, there exist some $\boldsymbol{\Sigma}_{\alpha}^{0}$-complete and some $\boldsymbol{\Pi}_{\alpha}^{0}$-complete infinitary rational relations accepted by 2 -tape Büchi automata. This surprising result gives answers to questions of Simonnet Sim92 and of Lescow and Thomas [Tho90, LT94].
\end{abstract}

Keywords: 2-tape Büchi automata; infinitary rational relations; Cantor topology; topological complexity; Borel hierarchy; complete sets.

\section{Introduction}

In the sixties, automata accepting infinite words were firstly considered by Büchi in order to study decidability of the monadic second order theory S1S of one successor over the integers Büc62]. Then the so called $\omega$-regular languages have been intensively studied and have found many applications for specification and verification of non terminating systems, see [Tho90, Sta97, PP04] for many results and references. On the other hand, rational relations on finite words were also studied in the sixties, and played a fundamental role in the study of families of context free languages Ber79]. Investigations on their extension to rational relations on infinite words were carried out or mentioned in the books BT70.LS77]. Gire and Nivat studied infinitary rational relations in Gir81,GN84. These relations are sets of pairs of infinite words which are accepted by 2 -tape finite Büchi automata with asynchronous reading heads. The class of infinitary rational relations, which extends both the class of finitary rational relations and the class of $\omega$-regular languages, and the rational functions they may define, have been much studied, see for example CG99,BCPS00,Sim92, Sta97, Pri00.

Notice that a rational relation $R \subseteq \Sigma_{1}^{\omega} \times \Sigma_{2}^{\omega}$ may be seen as an $\omega$-language over the alphabet $\Sigma_{1} \times \Sigma_{2}$.

A way to study the complexity of languages of infinite words accepted by finite machines is to study their topological complexity and firstly to locate them with regard to the Borel and the projective hierarchies. This work is analysed 
for example in [Sta86, Tho90, EH93, LT94,Sta97. It is well known that every $\omega$ language accepted by a Turing machine with a Büchi or Muller acceptance condition is an analytic set and that $\omega$-regular languages are boolean combinations of $\boldsymbol{\Pi}_{2}^{0}$-sets hence $\boldsymbol{\Delta}_{3}^{0}$-sets, Sta97,PP04.

The question of the topological complexity of relations on infinite words also naturally arises and is asked by Simonnet in Sim92]. It is also posed in a more general form by Lescow and Thomas in [LT94] (for infinite labelled partial orders) and in Tho89 where Thomas suggested to study reducibility notions and associated completeness results.

Every infinitary rational relation is an analytic set. We showed in Fin03a that there exist some infinitary rational relations which are analytic but non Borel, and in [Fin03c] that there are some $\boldsymbol{\Sigma}_{3}^{0}$-complete and some $\boldsymbol{\Pi}_{3}^{0}$-complete infinitary rational relations, using a coding of $\omega^{2}$-words by pairs of infinite words. Using a different coding we proved in [Fin03d that there exist such infinitary rational relations which have a very simple structure and can be easily described by their sections. Using this very simple structure, we constructed also some infinitary rational relations, accepted by 3 -tape Büchi automata, which are $\boldsymbol{\Sigma}_{4^{-}}^{0}$ complete.

On the other hand we recently proved in Fin05a Fin05b that the Borel hierarchy of $\omega$-languages accepted by Büchi real time 1-counter automata is equal to the Borel hierarchy of $\omega$-languages accepted by Büchi Turing machines. In particular, for each non null recursive ordinal $\alpha$, there exist some $\boldsymbol{\Sigma}_{\alpha}^{0}$-complete and some $\boldsymbol{\Pi}_{\alpha}^{0}$-complete $\omega$-languages accepted by Büchi real time 1-counter automata.

Using a simulation of real time 1-counter automata we prove in this paper a similar result: the Borel hierarchy of the class of infinitary rational relations is equal to the Borel hierarchy of $\omega$-languages accepted by Büchi real time 1-counter automata which is also equal to the Borel hierarchy of $\omega$-languages accepted by Büchi Turing machines. In particular, for each non null recursive ordinal $\alpha$, there exist some $\boldsymbol{\Sigma}_{\alpha}^{0}$-complete and some $\boldsymbol{\Pi}_{\alpha}^{0}$-complete infinitary rational relations. This gives answers to questions of Simonnet Sim92] and of Lescow and Thomas Tho90,LT94.

The paper is organized as follows. In section 2 we recall the notion of 2-tape automata and of real time 1-counter automata with Büchi acceptance condition. In section 3 we recall definitions of Borel and analytic sets, and we prove our main result in section 4 .

\section{2-tape automata and 1-counter automata}

We assume the reader to be familiar with the theory of formal $(\omega)$-languages [Tho90,Sta97]. We shall use usual notations of formal language theory.

When $\Sigma$ is a finite alphabet, a non-empty finite word over $\Sigma$ is any sequence $x=a_{1} \ldots a_{k}$, where $a_{i} \in \Sigma$ for $i=1, \ldots, k$, and $k$ is an integer $\geq 1$. The length of $x$ is $k$, denoted by $|x|$. The empty word has no letter and is denoted by $\lambda$; its length is 0 . For $x=a_{1} \ldots a_{k}$, we write $x(i)=a_{i}$ and $x[i]=x(1) \ldots x(i)$ for $i \leq k$ 
and $x[0]=\lambda . \Sigma^{\star}$ is the set of finite words (including the empty word) over $\Sigma$. The first infinite ordinal is $\omega$. An $\omega$-word over $\Sigma$ is an $\omega$-sequence $a_{1} \ldots a_{n} \ldots$, where for all integers $i \geq 1, \quad a_{i} \in \Sigma$. When $\sigma$ is an $\omega$-word over $\Sigma$, we write $\sigma=\sigma(1) \sigma(2) \ldots \sigma(n) \ldots$, where for all $i, \sigma(i) \in \Sigma$, and $\sigma[n]=\sigma(1) \sigma(2) \ldots \sigma(n)$ for all $n \geq 1$ and $\sigma[0]=\lambda$.

The prefix relation is denoted $\sqsubseteq$ : a finite word $u$ is a prefix of a finite word $v$ (respectively, an infinite word $v$ ), denoted $u \sqsubseteq v$, if and only if there exists a finite word $w$ (respectively, an infinite word $w$ ), such that $v=u$.w. The set of $\omega$-words over the alphabet $\Sigma$ is denoted by $\Sigma^{\omega}$. An $\omega$-language over an alphabet $\Sigma$ is a subset of $\Sigma^{\omega}$. The complement (in $\Sigma^{\omega}$ ) of an $\omega$-language $V \subseteq \Sigma^{\omega}$ is $\Sigma^{\omega}-V$, denoted $V^{-}$.

Infinitary rational relations are subsets of $\Sigma^{\omega} \times \Gamma^{\omega}$, where $\Sigma$ and $\Gamma$ are finite alphabets, which are accepted by 2-tape Büchi automata (2-BA).

Definition 1. A 2-tape Büchi automaton is a sextuple $\mathcal{T}=\left(K, \Sigma, \Gamma, \Delta, q_{0}, F\right)$, where $K$ is a finite set of states, $\Sigma$ and $\Gamma$ are finite alphabets, $\Delta$ is a finite subset of $K \times \Sigma^{\star} \times \Gamma^{\star} \times K$ called the set of transitions, $q_{0}$ is the initial state, and $F \subseteq K$ is the set of accepting states.

A computation $\mathcal{C}$ of the 2-tape Büchi automaton $\mathcal{T}$ is an infinite sequence of transitions

$$
\left(q_{0}, u_{1}, v_{1}, q_{1}\right),\left(q_{1}, u_{2}, v_{2}, q_{2}\right), \ldots\left(q_{i-1}, u_{i}, v_{i}, q_{i}\right),\left(q_{i}, u_{i+1}, v_{i+1}, q_{i+1}\right), \ldots
$$

The computation is said to be successful iff there exists a final state $q_{f} \in F$ and infinitely many integers $i \geq 0$ such that $q_{i}=q_{f}$.

The input word of the computation is $u=u_{1} \cdot u_{2} \cdot u_{3} \ldots$

The output word of the computation is $v=v_{1} \cdot v_{2} . v_{3} \ldots$

Then the input and the output words may be finite or infinite.

The infinitary rational relation $R(\mathcal{T}) \subseteq \Sigma^{\omega} \times \Gamma^{\omega}$ accepted by the 2-tape Büchi automaton $\mathcal{T}$ is the set of couples $(u, v) \in \Sigma^{\omega} \times \Gamma^{\omega}$ such that $u$ and $v$ are the input and the output words of some successful computation $\mathcal{C}$ of $\mathcal{T}$.

The set of infinitary rational relations will be denoted $R A T_{\omega}$.

Definition 2. A (real time) 1-counter machine is a 4-tuple $\mathcal{M}=\left(K, \Sigma, \Delta, q_{0}\right)$, where $K$ is a finite set of states, $\Sigma$ is a finite input alphabet, $q_{0} \in K$ is the initial state, and the transition relation $\Delta$ is a subset of $K \times \Sigma \times\{0,1\} \times K \times\{0,1,-1\}$. If the machine $\mathcal{M}$ is in state $q$ and $c \in \mathbf{N}$ is the content of the counter then the configuration (or global state) of $\mathcal{M}$ is $(q, c)$.

For $a \in \Sigma, q, q^{\prime} \in K$ and $c \in \mathbf{N}$, if $\left(q, a, i, q^{\prime}, j\right) \in \Delta$ where $i=0$ if $c=0$ and $i=1$ if $c \neq 0$ then we write:

$$
a:(q, c) \mapsto \mathcal{M}\left(q^{\prime}, c+j\right)
$$

$\mapsto_{\mathcal{M}}^{\star}$ is the transitive and reflexive closure of $\mapsto \mathcal{M}$.

Thus we see that the transition relation must satisfy: 
if $\left(q, a, i, q^{\prime}, j\right) \in \Delta$ and $i=0$ then $j=0$ or $j=1$ (but $j$ may not be equal to $-1)$.

Let $\sigma=a_{1} a_{2} \ldots a_{n}$ be a finite word over $\Sigma$. A sequence of configurations $r=$ $\left(q_{i}, c_{i}\right)_{1 \leq i \leq n+1}$ is called a run of $\mathcal{M}$ on $\sigma$, starting in configuration $(p, c)$, iff:

(1) $\left(q_{1}, c_{1}\right)=(p, c)$

(2) for each $i \in[1, n], a_{i}:\left(q_{i}, c_{i}\right) \mapsto \mathcal{M}\left(q_{i+1}, c_{i+1}\right)$

Let $\sigma=a_{1} a_{2} \ldots a_{n} \ldots$ be an $\omega$-word over $\Sigma$. An $\omega$-sequence of configurations $r=\left(q_{i}, c_{i}\right)_{i \geq 1}$ is called a run of $\mathcal{M}$ on $\sigma$, starting in configuration $(p, c)$, iff:

(1) $\left(q_{1}, c_{1}\right)=(p, c)$

(2) for each $i \geq 1, a_{i}:\left(q_{i}, c_{i}\right) \mapsto \mathcal{M}\left(q_{i+1}, c_{i+1}\right)$

For every such run, $\operatorname{In}(r)$ is the set of all states entered infinitely often during run $r$.

A run $r$ of $M$ on $\sigma$, starting in configuration $\left(q_{0}, 0\right)$, will be simply called " $a$ run of $M$ on $\sigma "$.

Definition 3. A (real time) Büchi 1-counter automaton is a 5-tuple

$$
\mathcal{M}=\left(K, \Sigma, \Delta, q_{0}, F\right),
$$

where $\mathcal{M}^{\prime}=\left(K, \Sigma, \Delta, q_{0}\right)$ is a (real time) 1-counter machine and $F \subseteq K$ is the set of accepting states. The $\omega$-language accepted by $\mathcal{M}$ is

$$
L(\mathcal{M})=\left\{\sigma \in \Sigma^{\omega} \mid \text { there exists a run } r \text { of } \mathcal{M} \text { on } \sigma \text { such that } \operatorname{In}(r) \cap F \neq \emptyset\right\}
$$

The class of (real time) Büchi 1-counter automata will be denoted r-BC(1).

The class of $\omega$-languages accepted by real time Büchi 1-counter automata will be denoted $\mathbf{r}-\mathbf{B C L}(1)_{\omega}$.

\section{Borel hierarchy}

We assume the reader to be familiar with basic notions of topology which may be found in Mos80, LT94, Kec95, Sta97,PP04. There is a natural metric on the set $\Sigma^{\omega}$ of infinite words over a finite alphabet $\Sigma$ which is called the prefix metric and defined as follows. For $u, v \in \Sigma^{\omega}$ and $u \neq v$ let $\delta(u, v)=2^{-l_{\operatorname{pref}(u, v)}}$ where $l_{\text {pref }(u, v)}$ is the first integer $n$ such that the $(n+1)^{s t}$ letter of $u$ is different from the $(n+1)^{s t}$ letter of $v$. This metric induces on $\Sigma^{\omega}$ the usual Cantor topology for which open subsets of $\Sigma^{\omega}$ are in the form $W \cdot \Sigma^{\omega}$, where $W \subseteq \Sigma^{\star}$. A set $L \subseteq \Sigma^{\omega}$ is a closed set iff its complement $\Sigma^{\omega}-L$ is an open set. Define now the Borel Hierarchy of subsets of $\Sigma^{\omega}$ :

Definition 4. For a non-null countable ordinal $\alpha$, the classes $\boldsymbol{\Sigma}_{\alpha}^{0}$ and $\boldsymbol{\Pi}_{\alpha}^{0}$ of the Borel Hierarchy on the topological space $\Sigma^{\omega}$ are defined as follows:

$\boldsymbol{\Sigma}_{1}^{0}$ is the class of open subsets of $\Sigma^{\omega}, \mathbf{\Pi}_{1}^{0}$ is the class of closed subsets of $\Sigma^{\omega}$, and for any countable ordinal $\alpha \geq 2$ :

$\boldsymbol{\Sigma}_{\alpha}^{0}$ is the class of countable unions of subsets of $\Sigma^{\omega}$ in $\bigcup_{\gamma<\alpha} \boldsymbol{\Pi}_{\gamma}^{0}$.

$\boldsymbol{\Pi}_{\alpha}^{0}$ is the class of countable intersections of subsets of $\Sigma^{\omega}$ in $\bigcup_{\gamma<\alpha} \Sigma_{\gamma}^{0}$. 
For a countable ordinal $\alpha$, a subset of $\Sigma^{\omega}$ is a Borel set of rank $\alpha$ iff it is in $\boldsymbol{\Sigma}_{\alpha}^{0} \cup \boldsymbol{\Pi}_{\alpha}^{0}$ but not in $\bigcup_{\gamma<\alpha}\left(\boldsymbol{\Sigma}_{\gamma}^{0} \cup \boldsymbol{\Pi}_{\gamma}^{0}\right)$.

There are also some subsets of $\Sigma^{\omega}$ which are not Borel. In particular the class of Borel subsets of $\Sigma^{\omega}$ is strictly included into the class $\Sigma_{1}^{1}$ of analytic sets which are obtained by projection of Borel sets, see for example Sta97, LT94,PP04,Kec95 for more details. The (lightface) class $\Sigma_{1}^{1}$ of effective analytic sets is the class of sets which are obtained by projection of arithmetical sets. It is well known that a set $L \subseteq \Sigma^{\omega}$, where $\Sigma$ is a finite alphabet, is in the class $\Sigma_{1}^{1}$ iff it is accepted by a Turing machine with a Büchi or Muller acceptance condition Sta97.

We now define completeness with regard to reduction by continuous functions. For a countable ordinal $\alpha \geq 1$, a set $F \subseteq \Sigma^{\omega}$ is said to be a $\boldsymbol{\Sigma}_{\alpha}^{0}$ (respectively, $\boldsymbol{\Pi}_{\alpha}^{0}, \boldsymbol{\Sigma}_{1}^{1}$ )-complete set iff for any set $E \subseteq Y^{\omega}$ (with $Y$ a finite alphabet): $E \in \boldsymbol{\Sigma}_{\alpha}^{0}$ (respectively, $E \in \boldsymbol{\Pi}_{\alpha}^{0}, E \in \boldsymbol{\Sigma}_{1}^{1}$ ) iff there exists a continuous function $f: Y^{\omega} \rightarrow$ $\Sigma^{\omega}$ such that $E=f^{-1}(F) . \boldsymbol{\Sigma}_{n}^{0}$ (respectively $\boldsymbol{\Pi}_{n}^{0}$ )-complete sets, with $n$ an integer $\geq 1$, are thoroughly characterized in Sta86].

\section{Topology and infinitary rational relations}

The first non-recursive ordinal, usually called the Church-Kleene ordinal, will be denoted below by $\omega_{1}^{\mathrm{CK}}$.

We have proved in Fin05a Fin05b the following result.

Theorem 5. For every non null countable ordinal $\alpha<\omega_{1}^{\mathrm{CK}}$, there exist some $\boldsymbol{\Sigma}_{\alpha}^{0}$-complete and some $\boldsymbol{\Pi}_{\alpha}^{0}$-complete $\omega$-languages in the class $\mathbf{r}$-BCL $(1)_{\omega}$.

We are going to prove a similar result for the class $R A T_{\omega}$, using a simulation of 1-counter automata.

We now first define a coding of an $\omega$-word over a finite alphabet $\Sigma$ by an $\omega$-word over the alphabet $\Gamma=\Sigma \cup\{A\}$, where $A$ is an additionnal letter not in $\Sigma$.

For $x \in \Sigma^{\omega}$ the $\omega$-word $h(x)$ is defined by :

$h(x)=A \cdot 0 \cdot x(1) \cdot A \cdot 0^{2} \cdot x(2) \cdot A \cdot 0^{3} \cdot x(3) \cdot A \cdot 0^{4} \cdot x(4) \cdot A \ldots \cdot A \cdot 0^{n} \cdot x(n) \cdot A \cdot 0^{n+1} \cdot x(n+1) \cdot A \ldots$

Then it is easy to see that the mapping $h$ from $\Sigma^{\omega}$ into $(\Sigma \cup\{A\})^{\omega}$ is continuous and injective.

Lemma 6. Let $\Sigma$ be a finite alphabet and $\alpha \geq 2$ be a countable ordinal. If $L \subseteq \Sigma^{\omega}$ is $\boldsymbol{\Pi}_{\alpha}^{0}$-complete (respectively, $\boldsymbol{\Sigma}_{\alpha}^{0}$-complete) then

$$
h(L) \cup h\left(\Sigma^{\omega}\right)^{-}
$$

is a $\boldsymbol{\Pi}_{\alpha}^{0}$-complete (respectively, $\boldsymbol{\Sigma}_{\alpha}^{0}$-complete) subset of $(\Sigma \cup\{A\})^{\omega}$. 
Proof. Let $L$ be a $\boldsymbol{\Pi}_{\alpha}^{0}$-complete (respectively, $\boldsymbol{\Sigma}_{\alpha}^{0}$-complete) subset of $\Sigma^{\omega}$, for some countable ordinal $\alpha \geq 2$.

The topological space $\Sigma^{\omega}$ is compact thus its image by the continuous function $h$ is also a compact subset of the topological space $(\Sigma \cup\{A\})^{\omega}$. The set $h\left(\Sigma^{\omega}\right)$ is compact hence it is a closed subset of $(\Sigma \cup\{A\})^{\omega}$ and its complement

$$
\left(h\left(\Sigma^{\omega}\right)\right)^{-}=(\Sigma \cup\{A\})^{\omega}-h\left(\Sigma^{\omega}\right)
$$

is an open (i.e. a $\left.\Sigma_{1}^{0}\right)$ subset of $(\Sigma \cup\{A\})^{\omega}$.

On the other side the function $h$ is also injective thus it is a bijection from $\Sigma^{\omega}$ onto $h\left(\Sigma^{\omega}\right)$. But a continuous bijection between two compact sets is an homeomorphism therefore $h$ induces an homeomorphism between $\Sigma^{\omega}$ and $h\left(\Sigma^{\omega}\right)$. By hypothesis $L$ is a $\boldsymbol{\Pi}_{\alpha}^{0}$ (respectively, $\boldsymbol{\Sigma}_{\alpha}^{0}$ )-subset of $\Sigma^{\omega}$ thus $h(L)$ is a $\boldsymbol{\Pi}_{\alpha}^{0}$ (respectively, $\boldsymbol{\Sigma}_{\alpha}^{0}$ )-subset of $h\left(\Sigma^{\omega}\right)$ (where Borel sets of the topological space $h\left(\Sigma^{\omega}\right)$ are defined from open sets as in the case of the topological space $\left.\Sigma^{\omega}\right)$.

The topological space $h\left(\Sigma^{\omega}\right)$ is a topological subspace of $(\Sigma \cup\{A\})^{\omega}$ and its topology is induced by the topology on $(\Sigma \cup\{A\})^{\omega}$ : open sets of $h\left(\Sigma^{\omega}\right)$ are traces on $h\left(\Sigma^{\omega}\right)$ of open sets of $(\Sigma \cup\{A\})^{\omega}$ and the same result holds for closed sets. Then one can easily show by induction that for every ordinal $\beta \geq 1, \boldsymbol{\Pi}_{\beta^{-}}^{0}$

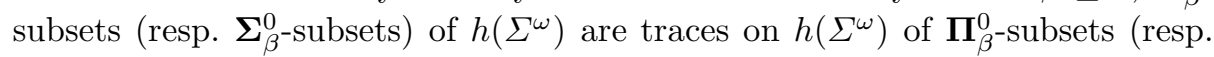
$\boldsymbol{\Sigma}_{\beta}^{0}$-subsets) of $(\Sigma \cup\{A\})^{\omega}$, i.e. are intersections with $h\left(\Sigma^{\omega}\right)$ of $\boldsymbol{\Pi}_{\beta}^{0}$-subsets (resp. $\boldsymbol{\Sigma}_{\beta}^{0}$-subsets $)$ of $(\Sigma \cup\{A\})^{\omega}$.

But $h(L)$ is a $\boldsymbol{\Pi}_{\alpha}^{0}$ (respectively, $\left.\boldsymbol{\Sigma}_{\alpha}^{0}\right)$-subset of $h\left(\Sigma^{\omega}\right)$ hence there exists a $\boldsymbol{\Pi}_{\alpha}^{0}$ (respectively, $\left.\boldsymbol{\Sigma}_{\alpha}^{0}\right)$-subset $T$ of $(\Sigma \cup\{A\})^{\omega}$ such that $h(L)=T \cap h\left(\Sigma^{\omega}\right)$. But $h\left(\Sigma^{\omega}\right)$ is a closed i.e. $\boldsymbol{\Pi}_{1}^{0}$-subset (hence also a $\boldsymbol{\Pi}_{\alpha}^{0}$ (respectively, $\boldsymbol{\Sigma}_{\alpha}^{0}$ )-subset) of $(\Sigma \cup\{A\})^{\omega}$ and the class of $\boldsymbol{\Pi}_{\alpha}^{0}$ (respectively, $\Sigma_{\alpha}^{0}$ )-subsets of $(\Sigma \cup\{A\})^{\omega}$ is closed under finite intersection thus $h(L)$ is a $\boldsymbol{\Pi}_{\alpha}^{0}$ (respectively, $\left.\boldsymbol{\Sigma}_{\alpha}^{0}\right)$-subset of $(\Sigma \cup\{A\})^{\omega}$.

Now $h(L) \cup\left(h\left(\Sigma^{\omega}\right)\right)^{-}$is the union of a $\boldsymbol{\Pi}_{\alpha}^{0}$ (respectively, $\left.\boldsymbol{\Sigma}_{\alpha}^{0}\right)$-subset and of a $\boldsymbol{\Sigma}_{1}^{0}$ subset of $(\Sigma \cup\{A\})^{\omega}$ therefore it is a $\boldsymbol{\Pi}_{\alpha}^{0}\left(\right.$ respectively, $\left.\boldsymbol{\Sigma}_{\alpha}^{0}\right)$-subset of $(\Sigma \cup\{A\})^{\omega}$ because the class of $\boldsymbol{\Pi}_{\alpha}^{0}$ (respectively, $\left.\boldsymbol{\Sigma}_{\alpha}^{0}\right)$-subsets of $(\Sigma \cup\{A\})^{\omega}$ is closed under finite union.

In order to prove that $h(L) \cup\left(h\left(\Sigma^{\omega}\right)\right)^{-}$is $\boldsymbol{\Pi}_{\alpha}^{0}$ (respectively, $\boldsymbol{\Sigma}_{\alpha}^{0}$ )--complete it suffices to remark that

$$
L=h^{-1}\left[h(L) \cup\left(h\left(\Sigma^{\omega}\right)\right)^{-}\right]
$$

This implies that $h(L) \cup\left(h\left(\Sigma^{\omega}\right)\right)^{-}$is $\boldsymbol{\Pi}_{\alpha}^{0}$ (respectively, $\boldsymbol{\Sigma}_{\alpha}^{0}$ )-complete because $L$ is assumed to be $\boldsymbol{\Pi}_{\alpha}^{0}$ (respectively, $\left.\boldsymbol{\Sigma}_{\alpha}^{0}\right)$-complete. 
Let now $\Sigma$ be a finite alphabet such that $0 \in \Sigma$ and let $\alpha$ be the $\omega$-word over the alphabet $\Sigma \cup\{A\}$ which is defined by:

$$
\alpha=A \cdot 0 \cdot A \cdot 0^{2} \cdot A \cdot 0^{3} \cdot A \cdot 0^{4} \cdot A \cdot 0^{5} \cdot A \ldots \cdot A \cdot 0^{n} \cdot A \cdot 0^{n+1} \cdot A \ldots
$$

We can now state the following Lemma.

Lemma 7. Let $\Sigma$ be a finite alphabet such that $0 \in \Sigma$, $\alpha$ be the $\omega$-word over $\Sigma \cup\{A\}$ defined as above, and $L \subseteq \Sigma^{\omega}$ be in $\mathbf{r}-\mathbf{B C L}(1)_{\omega}$. Then there exists an infinitary rational relation $R_{1} \subseteq(\Sigma \cup\{A\})^{\omega} \times(\Sigma \cup\{A\})^{\omega}$ such that:

$$
\forall x \in \Sigma^{\omega} \quad(x \in L) \text { iff }\left((h(x), \alpha) \in R_{1}\right)
$$

Proof. Let $\Sigma$ be a finite alphabet such that $0 \in \Sigma, \alpha$ be the $\omega$-word over $\Sigma \cup\{A\}$ defined as above, and $L=L(\mathcal{A}) \subseteq \Sigma^{\omega}$, where $\mathcal{A}=\left(K, \Sigma, \Delta, q_{0}, F\right)$ is a 1 -counter Büchi automaton.

We define now the relation $R_{1}$. A pair $y=\left(y_{1}, y_{2}\right)$ of $\omega$-words over the alphabet $\Sigma \cup\{A\}$ is in $R_{1}$ if and only if it is in the form

$y_{1}=A \cdot u_{1} \cdot v_{1} \cdot x(1) \cdot A \cdot u_{2} \cdot v_{2} \cdot x(2) \cdot A \cdot u_{3} \cdot v_{3} \cdot x(3) \cdot A \ldots A \cdot u_{n} \cdot v_{n} \cdot x(n) \cdot A \ldots$

$y_{2}=A \cdot w_{1} \cdot z_{1} \cdot A \cdot w_{2} \cdot z_{2} \cdot A \cdot w_{3} \cdot z_{3} \cdot A \ldots A \cdot w_{n} \cdot z_{n} \cdot A \ldots$

where $\left|v_{1}\right|=0$ and for all integers $i \geq 1$,

$$
\begin{gathered}
u_{i}, v_{i}, w_{i}, z_{i} \in 0^{\star} \text { and } x(i) \in \Sigma \text { and } \\
\left|u_{i+1}\right|=\left|z_{i}\right|+1
\end{gathered}
$$

and there is a sequence $\left(q_{i}\right)_{i \geq 0}$ of states of $K$ such that for all integers $i \geq 1$ :

$$
x(i):\left(q_{i-1},\left|v_{i}\right|\right) \mapsto_{\mathcal{A}}\left(q_{i},\left|w_{i}\right|\right)
$$

Moreover some state $q_{f} \in F$ occurs infinitely often in the sequence $\left(q_{i}\right)_{i \geq 0}$.

Notice that the state $q_{0}$ of the sequence $\left(q_{i}\right)_{i \geq 0}$ is also the initial state of $\mathcal{A}$.

Let now $x \in \Sigma^{\omega}$ such that $(h(x), \alpha) \in R_{1}$. We are going to prove that $x \in L$.

By hypothesis $(h(x), \alpha) \in R_{1}$ thus there are finite words $u_{i}, v_{i}, w_{i}, z_{i} \in 0^{\star}$ such that $\left|v_{1}\right|=0$ and for all integers $i \geq 1,\left|u_{i+1}\right|=\left|z_{i}\right|+1$, and

$h(x)=A \cdot u_{1} \cdot v_{1} \cdot x(1) \cdot A \cdot u_{2} \cdot v_{2} \cdot x(2) \cdot A \cdot u_{3} \cdot v_{3} \cdot x(3) \cdot A \ldots A \cdot u_{n} \cdot v_{n} \cdot x(n) \cdot A \ldots$

$\alpha=A \cdot w_{1} \cdot z_{1} \cdot A \cdot w_{2} \cdot z_{2} \cdot A \cdot w_{3} \cdot z_{3} \cdot A \ldots A \cdot w_{n} \cdot z_{n} \cdot A \ldots$

Moreover there is a sequence $\left(q_{i}\right)_{i \geq 0}$ of states of $K$ such that for all integers $i \geq 1$ :

$$
x(i):\left(q_{i-1},\left|v_{i}\right|\right) \mapsto_{\mathcal{A}}\left(q_{i},\left|w_{i}\right|\right)
$$


and some state $q_{f} \in F$ occurs infinitely often in the sequence $\left(q_{i}\right)_{i \geq 0}$.

on the other side we have:

$h(x)=A \cdot 0 \cdot x(1) \cdot A \cdot 0^{2} \cdot x(2) \cdot A \cdot 0^{3} \cdot x(3) \cdot A \ldots A \cdot 0^{n} \cdot x(n) \cdot A \cdot 0^{n+1} \cdot x(n+1) \cdot A \ldots$

$\alpha=A \cdot 0 \cdot A \cdot 0^{2} \cdot A \cdot 0^{3} \cdot A \cdot 0^{4} \cdot A \ldots A \cdot 0^{n} \cdot A \ldots$

So we have $\left|u_{1} \cdot v_{1}\right|=1$ and $\left|v_{1}\right|=0$ and $x(1):\left(q_{0},\left|v_{1}\right|\right) \mapsto_{\mathcal{A}}\left(q_{1},\left|w_{1}\right|\right)$. But $\left|w_{1} \cdot z_{1}\right|=1,\left|u_{2} \cdot v_{2}\right|=2$, and $\left|u_{2}\right|=\left|z_{1}\right|+1$ thus $\left|v_{2}\right|=\left|w_{1}\right|$.

We are going to prove in a similar way that for all integers $i \geq 1$ it holds that $\left|v_{i+1}\right|=\left|w_{i}\right|$.

We know that $\left|w_{i} \cdot z_{i}\right|=i,\left|u_{i+1} \cdot v_{i+1}\right|=i+1$, and $\left|u_{i+1}\right|=\left|z_{i}\right|+1$ thus $\left|w_{i}\right|=$ $\left|v_{i+1}\right|$.

Then for all $i \geq 1, x(i):\left(q_{i-1},\left|v_{i}\right|\right) \mapsto_{\mathcal{A}}\left(q_{i},\left|v_{i+1}\right|\right)$.

So if we set $c_{i}=\left|v_{i}\right|,\left(q_{i-1}, c_{i}\right)_{i \geq 1}$ is an accepting run of $\mathcal{A}$ on $x$ and this implies that $x \in L$.

Conversely it is easy to prove that if $x \in L$ then $(h(x), \alpha)$ may be written in the form of $\left(y_{1}, y_{2}\right) \in R_{1}$.

It remains to prove that the above defined relation $R_{1}$ is an infinitary rational relation. It is easy to find a 2 -tape Büchi automaton $\mathcal{T}$ accepting the infinitary rational relation $R_{1}$.

Lemma 8. The set

$$
R_{2}=(\Sigma \cup\{A\})^{\omega} \times(\Sigma \cup\{A\})^{\omega}-\left(h\left(\Sigma^{\omega}\right) \times\{\alpha\}\right)
$$

is an infinitary rational relation.

Proof. By definition of the mapping $h$, we know that a pair of $\omega$-words over the alphabet $(\Sigma \cup\{A\})$ is in $h\left(\Sigma^{\omega}\right) \times\{\alpha\}$ iff it is in the form $\left(\sigma_{1}, \sigma_{2}\right)$, where $\sigma_{1}=A \cdot 0 \cdot x(1) \cdot A \cdot 0^{2} \cdot x(2) \cdot A \cdot 0^{3} \cdot x(3) \cdot A \ldots . A \cdot 0^{n} \cdot x(n) \cdot A \cdot 0^{n+1} \cdot x(n+1) \cdot A .$. $\sigma_{2}=\alpha=A \cdot 0 \cdot A \cdot 0^{2} \cdot A \cdot 0^{3} \cdot A \ldots \cdot A \cdot 0^{n} \cdot A \cdot 0^{n+1} \cdot A \ldots$

where for all integers $i \geq 1, x(i) \in \Sigma$.

So it is easy to see that $(\Sigma \cup\{A\})^{\omega} \times(\Sigma \cup\{A\})^{\omega}-\left(h\left(\Sigma^{\omega}\right) \times\{\alpha\}\right)$ is the union of the sets $\mathcal{C}_{j}$ where:

- $\mathcal{C}_{1}$ is formed by pairs $\left(\sigma_{1}, \sigma_{2}\right)$ where

$\sigma_{1}$ has not any initial segment in $A \cdot \Sigma^{2} \cdot A \cdot \Sigma^{3} \cdot A$, or $\sigma_{2}$ has not any initial segment in A. $. \cdot A . \Sigma^{2} . A$.

$-\mathcal{C}_{2}$ is formed by pairs $\left(\sigma_{1}, \sigma_{2}\right)$ where $\sigma_{2} \notin\left(A .0^{+}\right)^{\omega}$, or $\sigma_{1} \notin\left(A .0^{+} . \Sigma\right)^{\omega}$. 
$-\mathcal{C}_{3}$ is formed by pairs $\left(\sigma_{1}, \sigma_{2}\right)$ where

$\sigma_{1}=A \cdot w_{1} \cdot A \cdot w_{2} \cdot A \cdot w_{3} \cdot A \ldots A \cdot w_{n} \cdot A \cdot u \cdot A \cdot z_{1}$

$\sigma_{2}=A \cdot w_{1}^{\prime} \cdot A \cdot w_{2}^{\prime} \cdot A \cdot w_{3}^{\prime} \cdot A \ldots A \cdot w_{n}^{\prime} \cdot A \cdot v \cdot A \cdot z_{2}$

where $n$ is an integer $\geq 1$, for all $i \leq n w_{i}, w_{i}^{\prime} \in \Sigma^{\star}, z_{1}, z_{2} \in(\Sigma \cup\{A\})^{\omega}$ and

$$
u, v \in \Sigma^{\star} \text { and }|u| \neq|v|+1
$$

$-\mathcal{C}_{4}$ is formed by pairs $\left(\sigma_{1}, \sigma_{2}\right)$ where

$\sigma_{1}=A \cdot w_{1} \cdot A \cdot w_{2} \cdot A \cdot w_{3} \cdot A \cdot w_{4} \ldots A \cdot w_{n} \cdot A \cdot w_{n+1} \cdot A \cdot v \cdot A \cdot z_{1}$

$\sigma_{2}=A \cdot w_{1}^{\prime} \cdot A \cdot w_{2}^{\prime} \cdot A \cdot w_{3}^{\prime} \cdot A \cdot w_{4}^{\prime} \ldots \cdot A \cdot w_{n}^{\prime} \cdot A \cdot u \cdot A \cdot z_{2}$

where $n$ is an integer $\geq 1$, for all $i \leq n w_{i}, w_{i}^{\prime} \in \Sigma^{\star}, w_{n+1} \in \Sigma^{\star}, z_{1}, z_{2} \in$ $(\Sigma \cup\{A\})^{\omega}$ and

$$
u, v \in \Sigma^{\star} \text { and }|v| \neq|u|+2
$$

Each set $\mathcal{C}_{j}, 1 \leq j \leq 4$, is easily seen to be an infinitary rational relation $\subseteq(\Sigma \cup\{A\})^{\omega} \times(\Sigma \cup\{A\})^{\omega}$ (the detailed proof is left to the reader). The class $R A T_{\omega}$ is closed under finite union thus

$$
R_{2}=(\Sigma \cup\{A\})^{\omega} \times(\Sigma \cup\{A\})^{\omega}-\left(h\left(\Sigma^{\omega}\right) \times\{\alpha\}\right)=\bigcup_{1 \leq j \leq 4} \mathcal{C}_{j}
$$

is an infinitary rational relation.

We can now state the following result :

Theorem 9. For every non null countable ordinal $\gamma<\omega_{1}^{\mathrm{CK}}$, there exists some $\boldsymbol{\Sigma}_{\gamma}^{0}$-complete and some $\boldsymbol{\Pi}_{\gamma}^{0}$-complete infinitary rational relations in the class $R A T_{\omega}$.

Proof. For $\gamma=1$ (and even $\gamma=2$ ) the result is already true for regular $\omega$ languages.

Let then $\gamma \geq 2$ be a countable non null recursive ordinal and $L=L(\mathcal{A}) \subseteq \Sigma^{\omega}$ be a $\boldsymbol{\Pi}_{\gamma}^{0}$-complete (respectively, $\boldsymbol{\Sigma}_{\gamma}^{0}$-complete) $\omega$-language accepted by a (real time) Büchi 1-counter automaton $\mathcal{A}$.

Let $\Gamma=\Sigma \cup\{A\}$ and $R_{1} \subseteq \Gamma^{\omega} \times \Gamma^{\omega}$ be the infinitary rational relation constructed from $L(\mathcal{A})$ as in the proof of Lemma 7 and let

$$
R=R_{1} \cup R_{2} \subseteq \Gamma^{\omega} \times \Gamma^{\omega}
$$

The class $R A T_{\omega}$ is closed under finite union therefore $R$ is an infinitary rational relation.

Lemma 0 and the definition of $R_{2}$ imply that $R_{\alpha}=\left\{\sigma \in \Gamma^{\omega} \mid(\sigma, \alpha) \in R\right\}$ is equal to the set $\mathcal{L}=h(L) \cup\left(h\left(\Sigma^{\omega}\right)\right)^{-}$which is a $\Pi_{\gamma^{0}}^{0}$-complete (respectively, $\Sigma_{\gamma}^{0}$-complete) subset of $(\Sigma \cup\{A\})^{\omega}$ by Lemma 6 . 
Moreover, for all $u \in \Gamma^{\omega}-\{\alpha\}, R_{u}=\left\{\sigma \in \Gamma^{\omega} \mid(\sigma, u) \in R\right\}=\Gamma^{\omega}$ holds by definition of $R_{2}$.

In order to prove that $R$ is a $\boldsymbol{\Pi}_{\gamma}^{0}$ (respectively, $\boldsymbol{\Sigma}_{\gamma}^{0}$ )-complete set remark first that $R$ may be written as the union:

$$
R=\mathcal{L} \times\{\alpha\} \quad \bigcup \Gamma^{\omega} \times\left(\Gamma^{\omega}-\{\alpha\}\right)
$$

We already know that $\mathcal{L}$ is a $\boldsymbol{\Pi}_{\gamma}^{0}$ (respectively, $\boldsymbol{\Sigma}_{\gamma}^{0}$ )-complete subset of ( $\Sigma \cup$ $\{A\})^{\omega}$. Then it is easy to show that $\mathcal{L} \times\{\alpha\}$ is also a $\boldsymbol{\Pi}_{\gamma}^{0}$ (respectively, $\boldsymbol{\Sigma}_{\gamma}^{0}$ )subset of $(\Sigma \cup\{A\})^{\omega} \times(\Sigma \cup\{A\})^{\omega}$. On the other side it is easy to see that $\Gamma^{\omega} \times\left(\Gamma^{\omega}-\{\alpha\}\right)$ is an open subset of $\Gamma^{\omega} \times \Gamma^{\omega}$. Thus $R$ is a $\Pi_{\gamma}^{0}$ (respectively, $\boldsymbol{\Sigma}_{\gamma}^{0}$ )-set because the Borel class $\boldsymbol{\Pi}_{\gamma}^{0}$ (respectively, $\boldsymbol{\Sigma}_{\gamma}^{0}$ ) is closed under finite union.

Moreover let $g: \Sigma^{\omega} \rightarrow(\Sigma \cup\{A\})^{\omega} \times(\Sigma \cup\{A\})^{\omega}$ be the function defined by:

$$
\forall x \in \Sigma^{\omega} \quad g(x)=(h(x), \alpha)
$$

It is easy to see that $g$ is continuous because $h$ is continuous. By construction it turns out that for all $\omega$-words $x \in \Sigma^{\omega}, \quad(x \in L)$ iff $((h(x), \alpha) \in R)$ iff $(g(x) \in R)$. This means that $g^{-1}(R)=L$. This implies that $R$ is $\boldsymbol{\Pi}_{\gamma}^{0}$ (respectively, $\boldsymbol{\Sigma}_{\gamma}^{0}$ )complete because $L$ is $\boldsymbol{\Pi}_{\gamma}^{0}$ (respectively, $\boldsymbol{\Sigma}_{\gamma}^{0}$ )-complete.

Remark 10. The structure of the infinitary rational relation $R$ can be described very simply by the sections $R_{u}, u \in \Gamma^{\omega}$. All sections but one are equal to $\Gamma^{\omega}$, so they have the lowest topological complexity and exactly one section $\left(R_{\alpha}\right)$ is $a \boldsymbol{\Pi}_{\gamma}^{0}$ (respectively, $\boldsymbol{\Sigma}_{\gamma}^{0}$ )-complete subset of $\Gamma^{\omega}$.

\section{Concluding remarks}

The Wadge hierarchy is a great refinement of the Borel hierarchy and we have proved in Fin05a, Fin05b that the Wadge hierarchy of the class $\mathbf{r}-\mathbf{B C L}(1)_{\omega}$ is equal to the Wadge hierarchy of the class of $\omega$-languages accepted by Büchi Turing machines. Using the above coding and similar reasoning as in Fin05b, we can easily infer that the Wadge hierarchy of the class $R A T_{\omega}$ and the Wadge hierarchy of the class $\mathbf{r}-\mathbf{B C L}(1)_{\omega}$ are equal. Thus the Wadge hierarchy of the class $R A T_{\omega}$ is also the Wadge hierarchy of the (lightface) class $\Sigma_{1}^{1}$ of $\omega$-languages accepted by Turing machines with a Büchi acceptance condition. In particular their Borel hierarchies are also equal.

We have to indicate here a mistake in Fin05a. We wrote in that paper that it is well known that if $L \subseteq \Sigma^{\omega}$ is a $\Sigma_{1}^{1}$ set (i.e. accepted by a Turing machine with a Büchi acceptance condition), and is a Borel set of rank $\alpha$, then $\alpha$ is smaller than $\omega_{1}^{\mathrm{CK}}$. This fact, which is true if we replace $\Sigma_{1}^{1}$ by $\Delta_{1}^{1}$, seemed to us an obvious fact, and was accepted by many people as true, but it is actually not true. Kechris, Marker and Sami proved in [KMS89] that the supremum of the 
set of Borel ranks of (lightface) $\Pi_{1}^{1}$, so also of (lightface) $\Sigma_{1}^{1}$, sets is the ordinal $\gamma_{2}^{1}$. This ordinal is defined in KMS89 and it is proved to be strictly greater than the ordinal $\delta_{2}^{1}$ which is the first non $\Delta_{2}^{1}$ ordinal. Thus it holds that $\omega_{1}^{\mathrm{CK}}<\gamma_{2}^{1}$.

The ordinal $\gamma_{2}^{1}$ is also the supremum of the set of Borel ranks of $\omega$-languages in the class $\mathbf{r}-\mathbf{B C L}(1)_{\omega}$ or in the class $R A T_{\omega}$. Notice however that it is not proved in KMS89 that every non null ordinal $\gamma<\gamma_{2}^{1}$ is the Borel rank of a (lightface) $\Pi_{1}^{1}$ (or $\left.\Sigma_{1}^{1}\right)$ set, while it is known that every ordinal $\gamma<\omega_{1}^{\mathrm{CK}}$ is the Borel rank of a (lightface) $\Delta_{1}^{1}$ set. The situation is then much more complicated than it could be expected. More details will be given in the full versions of [Fin05a and of this paper.

Acknowledgements. Thanks to the anonymous referees for useful comments on a preliminary version of this paper.

\section{References}

BT70. Ya M. Barzdin and B.A. Trakhtenbrot, Finite Automata, Behaviour and Synthesis, Nauka, Moscow, 1970 (English translation, North Holland, Amsterdam, 1973).

BC00. M.-P. Bal and O. Carton, Determinization of Transducers over Infinite Words, in ICALP'2000 (U. Montanari et al., eds.), vol. 1853 of Lect. Notes in Comput. Sci., pp. 561-570, 2000.

BCPS00. M.-P. Béal, O. Carton, C. Prieur and J. Sakarovitch, Squaring Transducers: An Efficient Procedure for Deciding Functionality and Sequentiality, Theoretical Computer Science, vol. 292, no. 1, pp. 45-63, 2003.

Ber79. J. Berstel, Transductions and Context Free Languages, Teubner Verlag, 1979.

Büc62. J.R. Büchi, On a Decision Method in Restricted Second Order Arithmetic, Logic Methodology and Philosophy of Science, ( Proc. 1960 Int. Congr. ), Stanford University Press, 1962, 1-11.

CDT02. T. Cachat, J. Duparc and W. Thomas, Solving Pushdown Games with a $\Sigma_{3}$ Winning Condition, proceedings of CSL 2002, Lecture Notes in Computer Science, Springer, Volume 2471, pp. 322-336,

Cho77. C. Choffrut, Une Caractérisation des Fonctions Séquentielles et des Fonctions Sous-Séquentielles en tant que Relations Rationnelles, Theoretical Computer Science, Volume 5, 1977, p.325-338.

CG99. C. Choffrut and S. Grigorieff, Uniformization of Rational Relations, Jewels are Forever 1999, J. Karhumki, H. Maurer, G. Paun and G. Rozenberg editors, Springer, p.59-71.

EH93. J. Engelfriet and H. J. Hoogeboom, X-automata on $\omega$-Words, Theoretical Computer Science, Volume 110, (1993) 1, 1-51.

Fin03a. O. Finkel, On the Topological Complexity of Infinitary Rational Relations, RAIRO-Theoretical Informatics and Applications, Volume 37 (2), 2003, p. 105113.

Fin03b. O. Finkel, Undecidability of Topological and Arithmetical Properties of Infinitary Rational Relations, RAIRO-Theoretical Informatics and Applications, Volume 37 (2), 2003, p. 115-126.

Fin03c. O. Finkel, On Infinitary Rational Relations and Borel Sets, in the Proceedings of the Fourth International Conference on Discrete Mathematics and Theoretical 
Computer Science DMTCS'03, 7 - 12 July 2003, Dijon, France, Lecture Notes in Computer Science, Springer, Volume 2731, p. 155-167.

Fin03d. O. Finkel, On Infinitary Rational Relations and the Borel Hierarchy, submitted to Logical Methods in Computer Science, 27 pages.

Fin05a. O. Finkel, Borel Ranks and Wadge Degrees of Context Free $\omega$-Languages, in the Proceedings of the First Conference on Computability in Europe: New Computational Paradigms, CiE 2005, Amsterdam, The Netherlands, Lecture Notes in Computer Science, Volume 3526, Springer, 2005, p. 129-138.

Fin05b. O. Finkel, Borel Ranks and Wadge Degrees of Context Free $\omega$-Languages, long version of [Fin05a], Mathematical Structures in Computer Science, Special Issue on Mathematics of Computation at CIE 2005, to appear.

Gir81. F. Gire, Relations Rationnelles Infinitaires, Thèse de troisième cycle, Université Paris 7, Septembre 1981.

Gir83. F. Gire, Une Extension aux Mots Infinis de la Notion de Transduction Rationnelle, 6th GI Conf., Lect. Notes in Comp. Sci., Volume 145, 1983, p. 123-139.

GN84. F. Gire and M. Nivat, Relations Rationnelles Infinitaires, Calcolo, Volume XXI, 1984, p. 91-125.

Kec95. A. S. Kechris, Classical Descriptive Set Theory, Springer-Verlag, 1995.

KMS89. A. S. Kechris, D. Marker, and R. L. Sami, $\Pi_{1}^{1}$ Borel Sets, The Journal of Symbolic Logic, Volume 54 (3), 1989, p. 915-920.

Kur66. K. Kuratowski, Topology, Academic Press, New York 1966.

Lan69. L. H. Landweber, Decision Problems for $\omega$-Automata, Math. Syst. Theory 3 (1969) 4,376-384.

LT94. H. Lescow and W. Thomas, Logical Specifications of Infinite Computations, In: "A Decade of Concurrency" (J. W. de Bakker et al., eds), Lecture Notes in Computer Science, Springer, Volume 803 (1994), 583-621.

LS77. R. Lindner and L. Staiger, Algebraische Codierungstheorie - Theorie der Sequentiellen Codierungen, Akademie-Verlag, Berlin, 1977.

Mos80. Y. N. Moschovakis, Descriptive Set Theory, North-Holland, Amsterdam 1980.

PP04. D. Perrin and J.-E. Pin, Infinite Words, Automata, Semigroups, Logic and Games, Volume 141 of Pure and Applied Mathematics, Elsevier, 2004.

Pin96. J-E. Pin, Logic, Semigroups and Automata on Words, Annals of Mathematics and Artificial Intelligence 16 (1996), p. 343-384.

Pri00. C. Prieur, Fonctions Rationnelles de Mots Infinis et Continuité, Thèse de Doctorat, Université Paris 7, Octobre 2000.

Sim92. P. Simonnet, Automates et Théorie Descriptive, Thèse de Doctorat, Université Paris 7, March 1992.

Sta86. L. Staiger, Hierarchies of Recursive $\omega$-Languages, Jour. Inform. Process. Cybernetics EIK 22 (1986) 5/6, 219-241.

Sta97. L. Staiger, $\omega$-Languages, Chapter of the Handbook of Formal languages, Vol 3, edited by G. Rozenberg and A. Salomaa, Springer-Verlag, Berlin.

SW78. L. Staiger and K. Wagner, Rekursive Folgenmengen I, Z. Math Logik Grundlag. Math. 24, 1978, 523-538.

Tho89. W. Thomas, Automata and Quantifier Hierarchies, in: Formal Properties of Finite automata and Applications, Ramatuelle, 1988, Lecture Notes in Computer Science 386, Springer, Berlin, 1989, p.104-119.

Tho90. W. Thomas, Automata on Infinite Objects, in: J. Van Leeuwen, ed., Handbook of Theoretical Computer Science, Vol. B (Elsevier, Amsterdam, 1990 ), p. 133-191. 